\title{
CHARACTERIZATION AND INVOLVEMENT OF TOLL AND IMD PATHWAYS IN AHPND-INFECTED SHRIMP
}

\author{
Mi-De Yeh, Han-Ching Wang ${ }^{\S}$ \\ Institute of Biotechnology, College of Bioscience and Biotechnology, National Cheng \\ Kung University, Tainan, Taiwan
}

\begin{abstract}
Acute hepatopancreatic necrosis disease (AHPND) is emerging as a major disease of shrimp industries worldwide, causing almost $100 \%$ mortality at the postlarvae stage. The etiology is Vibrio parahaemolyticus ( Vp), a Gram-negative marine bacteria, which must have received a plasmid coding for production of PirA/B, a protein toxin. Based on molecular and histological studies, $V p$ accumulated in the stomach and secreted PirA/B, which damaged the stomach and hepatopancreas (major AHPND lesions). However, the mechanism by which AHPND kills shrimp remains unclear. Here, we demonstrated that genes of Toll and IMD pathway, Toll, MyD88 and Relish, and its downstream antimicrobial peptides (AMPs) genes, PEN2, 3, 4, anti-lipopolysaccharide factor (ALF), crustin and lysozyme (lyz), were suppressed in stomach. Surprisingly, this phenomenon also occurred in hemocytes (major immune organ of shrimp). Furthermore, most of these genes were overexpressed (particularly in the IMD pathway), in the hepatopancreas, a target organ for AHPND. We subsequently used dsRNAmediated silencing to confirm involvement of Toll and IMD pathway in pathogenesis of
\end{abstract} AHPND.

\section{KEYWORDS}

Acute hepatopancreatic necrosis disease, Toll pathway, IMD pathway, immune suppression, L. vannamei,

§Corresponding author. TEL: +886-6-2757575 ext 65603-810 FAX: +886-6-276-6505

E-mail: wanghc@mail.ncku.edu.tw 